\title{
Relación de obesidad y cirugía bariátrica
}

\section{Relationship between obesity and bariatric surgery}

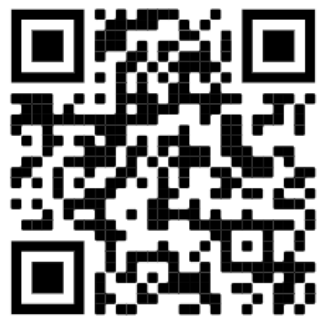

Recibido $15 / 08 / 2020$

\author{
${ }^{1}$ Dr. John Alejandro Solano Hidalgo \\ Investigador independiente, San José, Costa Rica \\ (D) https://orcid.org/0000-0001-6563-1178
}

${ }^{2}$ Dra. Raquel Sofía Ramírez Fallas Investigadora independiente, San José, Costa Rica

(iD) https://orcid.org/0000-0003-1717-4771

${ }^{3} \mathrm{Dr}$. Rodolfo José Alfaro Pacheco Investigador independiente, San José, Costa Rica

(iD) https://orcid.org/0000-0003-0631-2243

Corregido $15 / 10 / 2020$

Aceptado 20/10/2020

\section{RESUMEN}

La obesidad se define como un estado patológico que se caracteriza por un exceso de grasa en el cuerpo y es un problema de salud a nivel mundial el cual ha ido en aumento y se ha convertido en uno de los principales factores de riesgo para enfermedades cardiovasculares y endocrinológicas. El índice de masa corporal es un indicador del balance entre peso y estatura de cada individuo y es de gran utilidad para determinar estados nutricionales e identificar los pacientes de alto riesgo de desarrollar enfermedades por esta comorbilidad. Existen diferentes estrategias de tratamiento para reducción de obesidad, entre las cuales, las intervenciones quirúrgicas han demostrado un reducción importante en el peso corporal, así como un mantenimiento del mismo.

xccxcxc

PALABRAS CLAVE: obesidad; índice masa corporal; cirugía bariátrica; sobrepeso; gastrectomía.

\section{ABSTRACT}

Obesity is defined as a pathological state that is characterized by an excess of fat in the body and is a global health problem which has been increasing and has become one of the main risk factors for cardiovascular diseases and endocrinological. The body mass index is an indicator of the balance between weight and height of each individual and is very useful to determine nutritional states and identify patients at high risk of developing diseases due to this comorbidity. There are different treatment strategies for obesity reduction, among which, surgical interventions have shown a significant reduction in body weight, as well as its maintenance.

KEYWORDS: obesity; body mass index; bariatric surgery; overweight; gastrectomy.

\footnotetext{
${ }^{1}$ Médico general, graduado de la Universidad Ciencias Médicas (UCIMED), cod. MED16589, correo: john Solano@hotmail.com

${ }^{2}$ Médica general, graduado de la Universidad Ciencias Médicas (UCIMED), cod. MED16594, correo: doctoraramirezf@gmail.com

${ }^{3}$ Médico general, graduado de la Universidad Ciencias Médicas (UCIMED), cod. MED16029, correo: rodolfoalfaro0692@gmail.com
} 


\section{INTRODUCCIÓN}

El término obesidad se refiere al exceso de grasa en el cuerpo, la cual se mide normalmente por la relación entre peso corporal y la estatura (antropometría. Sin embargo, se observa un aumento drástico en la prevalencia de la obesidad, así como un riesgo mayor en las enfermedades cardiovasculares. El índice de masa corporal (IMC) es un método estandarizado aceptado para clasificación del peso corporal en niños mayores a 2 años, adolescentes y adultos. EI IMC provee una relación directa entre el peso en kilogramos y la estatura en metros $(1,2)$.

La obesidad a menudo se estigmatiza y conlleva una falsa percerepción que la causa principal es la falta de voluntad que conduce a elecciones dietéticas inapropiadas e inactividad física. Sin embargo, existe evidencia clínica que presenta la obesidad como una complicada condición médica crónica causada por la interacción de múltiples genéticos, ambientales, factores metabólicos y de comportamiento. Se describen múltiples tratamientos para esta patología, sin embargo, se pretende mediante esta revisión efectuar una síntesis de información actualizada que englobe la utilidad de la cirugía bariátrica como estrategia para una disminución rápida de peso como la mejor opción en estadios avanzados de obesidad (2-4).

\section{MÉTODOS}

Para la redacción de esta revisión se realizó una búsqueda de artículos y publicaciones científicas en bases de datos como: The New England Journal of Medicine, PubMed, Scielo, UpToDate y Google Scholar utilizando palabras claves como "Obesidad, "Cirugía bariátrica", "Gastrectomía". Se tomaron en cuenta únicamente artículos en idiomas inglés y español, los cuales estuvieran indexados en revistas digitales con un Digital Object Identifier System (DOI). Todos los artículos utilizados fueron publicados en el período 2015-2020. En total se recopilaron 18 artículos de los cuales solamente se utilizaron 16, se excluyó por el año de publicación e información contemplada.

\section{EPIDEMIOLOGÍA}

La obesidad es una enfermedad crónica que ha ido en aumento. En el 2015 aproximadamente 108 millones de niños y 604 millones de agultos globlamente eran obesos, esto representa un aumento casi del doble en prevalencia de obesidad en casi todos los países desde 1980 (3).

A nivel global, el IMC va en aumento. En países desarrollados la obesidad se encuentra en $18 \%$ y $20 \%$ de los hombres y mujeres respectivamente. A pesar de rango de países los datos sugieren que el porcentaje de obesidad ha incrementado en los últimos 30 años. En las últimas 3 décadas y media la prevalencia de obesidad ha aumentado el double mundialmente, adultos mayores de 18 años o más, con $11 \%$ en hombres y $15 \%$ de mujeres eran obesos en el 2015. Más de 42 millones de niños menores de cinco años se encontraban con sobrepeso en el $2014(3,4)$.

\section{DEFINICIÓN}

El índice de masa corporal es la medida común utilizada en la práctica clínica para la captación de obesidad. El peso y altura son necesarios para determiner el IMC, el cual se calcula con el peso en kilogramos dividido por la altura en metros cuadrados $(\mathrm{kg} / \mathrm{m} 2)$. Sin embargo, esta definición depende del 
método usado para determinar la presencia de obesidad (IMC o circunferencia abdominal) (1).

Para este artículo se utilizará la clasificación de obesidad con base en la Organización Mundial de la Salud (TABLA 1). No existe una tabla de consenso internacional de IMC en niños, sin embargo en esta población se clasifica como obesidad cuando el peso para su edad y sexo se encuentra por encima del percentil 95 basado en las gráficas de Control de Enfermedad y Prevención de Crecimiento $(1,11)$. Los cortes del IMC no estiman el riesgo cardiovascular asociado con obesidad, como lo es el caso de diabetes e hipertensión arterial, por lo que es fundamental captar y valorar un paciente con IMC elevado y determinar el riesgo cardiovascular. La cirunferencia abdominal, en adición a la medida del IMC se recomienda medir en pacientes con sobre peso $u$ obesidad. Una circunferencia abdominal $\geq 102 \mathrm{~cm}$ para hombre $\mathrm{y} \geq 88 \mathrm{~cm}$ en mujeres se considera como un factor de alto riesgo cardiovascular, ya que la circunferencia abdominal es el predictor de mortalidad en pacientes obesos.

Una medición de circunferencia abdominal en pacientes con IMC $\geq 35 \mathrm{~kg} / \mathrm{m}$ es innecesaria, estos pacientes ya tienen un alto riesgo proveniente de su adiposidad. Los pacientes con obesidad abdominal (central, visceral o androide) tiene un riesgo elevado de enfermedad cardíaca, diabetes, hipertensión, dislipidemia y de esteatosis hepática no alcohólica (2-4).

\section{MECANISMOS DE OBESIDAD}

La obesidad es una enfermedad la cual presenta un aumento drástico en las últimas décadas, la cual es causada por distintos factores como el ambiente, factores humorales, genéticos y combinación entre estos. Los factores ambientales contribuyen a un aumento de la obesidad, no obstante, no son limitantes en los estilos de vida adquiridos. Existen factores adquiridos como lo son disminución en actividad física, aumento de tiempo de televisión, estilo de

TABLA 1. Clasificación internacional del adulto con bajo peso, sobre peso u obesidad de acuerdo con el índice de masa corporal

\begin{tabular}{|c|c|}
\hline Clasificación & IMC $\left(\mathrm{kg} / \mathrm{m}^{2}\right)$ \\
\hline Bajo peso severo & $<18.50 \mathrm{~kg} / \mathrm{m}^{2}$ \\
\hline Bajo peso moderado & $<16.00 \mathrm{~kg} / \mathrm{m}^{2}$ \\
\hline Bajo peso leve & $16.00-16.99 \mathrm{~kg} / \mathrm{m}^{2}$ \\
\hline Rango normal & $18.50-24.99 \mathrm{~kg} / \mathrm{m}^{2}$ \\
\hline Sobrepeso & $\geq 25.00 \mathrm{~kg} / \mathrm{m}^{2}$ \\
\hline Pre-obeso & $25.00-29.99 \mathrm{~kg} / \mathrm{m}^{2}$ \\
\hline Obesidad & $\geq 30.00 \mathrm{~kg} / \mathrm{m}^{2}$ \\
\hline Obesidad: clase I & $30.00-34.99 \mathrm{~kg} / \mathrm{m}^{2}$ \\
\hline Obesidad: clase II & $35.00-39.99 \mathrm{~kg} / \mathrm{m}^{2}$ \\
\hline Obesidad: clase III & $\geq 40.00 \mathrm{~kg} / \mathrm{m}^{2}$ \\
\hline Fuente: Organización Mundial de la Salud: Clasificación IMC 2016 & \\
\hline
\end{tabular}


vida sedentaria, aumento de consumo de alimento con alto contenido calórico, aumentando porciones de comida y el uso de medicamentos que producen aumento de peso como efecto secundario. Sin embargo, aunque individuos estén expuestos a estos factores ambientales, no todos desarrollarán obesidad, ya que existen diferentes mecanismos que predisponen a ciertos individuos a desarrollar obesidad $(3,4)$. Muchos genes se han identificado como potenciales contribuyentes a desarrollar obesidad, posiblemente actuando en combinación.

Estudios en gemelos han demostrado una alta herencia de comportamientos alimentarios (53\%-84\%) $(6,7)$. Uno de los más estudiados el de masa grasa y el gen asociado a obesidad el cual se ha visto que se modifica con estilos de vida saludables (8).

Relativamente pocos individuos tiene formas monogénicas de obesidad, aunque más de 200 tipos de genes únicos mutados se han encontrado causantes de obesidad.

El hipotálamo regula la homeostasis de energía e integra señales hormonales de la periferia y comunica con el resto del sistema nervioso central. La leptina se secreta por el tejido adiposo y circula proporcionalmente a la cantidad de masa grasa correspondiente (8).

En bajos niveles de masa grasa; la leptina circula con parámetros bajos y se comunica con neuronas del hipotálamo para aumentar consumo de energía. En un estado de obesidad, ocurre lo opuesto y la leptina circula en altos niveles, sin embargo, los niveles altos de leptina, no aumenta el consumo de energía por un estado de resistencia a leptina, demostrando un estado de resistencia a la homeostasis del control de alimentación $(8,9)$.
A parte del sistema homeostático, el sistema neuronal es más potente en términos de regulación de apetito y obesidad. La comida es naturalmente gratificante y este sistema puede estar alterado en pacientes con obesidad, llevando a un estado exacerbado de aumento de peso (9).

Las dos teorías primarias corresponden a una hiporespuesta a los mecanismos de satisfacción que conllevan a una búsqueda con mayor satisfacción a los alimentos con alto contenido graso y calórico; por otra parte, también se plantea la hiperrespuesta a los aliemtos que conlleva a individuos a aumenta la ingesta calórica. Estas dos teorías se sustentan de la observación de respuesta a nivel de receptores dopaminérgicos "D2" en individuos con obesidad (10).

Las emociones también son potentes reguladores del apetito porque un ánimo depresivo y ansiedad son comorbilidades para desarrollar obesidad; relacionada con obesidad central en particular. Estrés es conocido por provocar cambios en apetito los cuales pueden conducir a desarrollar obesidad $(9,10)$.

\section{SÍNDROME METABÓLICO EN RELACIÓN CON OBESIDAD}

El aumento del tejido adiposo se relaciona con cuatro mecanismos; el primero; con aumento de producción de lípidos, triglicéridos y liberación de ácidos grasos, fenómenos que se relacionan con lipotixicidad y dislipidemia los cuales conllevan a largo plazo a esteatosis hepática no alcohólica y/o cirrosis, así como la dislipidemia conlleva a un alto riesgo de enfermedad arterial coronaria.

De segundo mecanismo, se encuentra el aumento de la actividad simpática y aumento 
del sistema renina, angiotensina, aldosterona lo que conlleva a un aumento de la presión pulmonar y sistémica.

De tercer mecanismo se encuentra el aumento de citoquinas proinflamatorias que conllevan a procesos como infertilidad, síndrome de ovario poliquístico, cáncer y un desbalance en la respuesta a la insulina y resistencia a la misma, conllevando a Diabetes Mellitus tipo dos.

Finalmente, de cuarto proceso, el aumento de peso por mecanismos de estrés (aumento de compresión renal, aumento de tejido blando en cuello, articulaciones y el aumento de la presión intra-adbominal) los cuales conllevan a aumento de presión pulmonar y sistémica, apnea obstructiva del sueño, osteoartrosis, reflujo gastroesofágico, cáncer esofágico y enfermedad coronaria.

Todos estos mecanismos conllevan a un aumento de incidencia de insuficiencia cardíaca congestiva, enfermedad cerebral y enfermedad vasculares (11-13).

\section{EFECTOS DE PÉRDIDA DE PESO}

La pérdida de peso de $5 \mathrm{~kg}$ se asocia con una reducción de más del $50 \%$ de riesgo de desarrollar diabetes mellitus. Produce una reducción del $15 \%$ de niveles de glucosa en ayunas y una disminución del $7 \%$ en los niveles de hemoglobina glicosilada (HbA1c) (13).

La disminución de peso de aproximadamente $10 \mathrm{~kg}$ se asocia con reducción del $20 \%$ de presión arterial sistólica y diastólica. Una mejoría similar del perfil lipídico de los pacientes con una disminución de lipoproteínas de baja densidad (LDL) de 1\%, triglicéridos (TG) de un 3\% y un incremento de lipoproteínas de alta densidad (HDL) de $1 \%$, por cada kilogramo de peso disminuido (13-15).

\section{CIRUGÍA BARIÁTRICA}

Los procedimientos de cirugía bariátrica se asocian con una pérdida de peso mayor en comparación con otras intervenciones, como cambios en estilo de vida y farmacoterapia. Según guías de la Sociedad Americana de Cirugía Bariátrica y metabólica, recomiendan la cirugía bariátrica a pacientes con IMC superior a $40 \mathrm{~kg} / \mathrm{m}^{2}$ y pacientes con IMC superior a $35 \mathrm{~kg} / \mathrm{m}^{2}$ y comorbilidades que puedan ser mejoradas con pérdida de peso $(6,11)$.

Los pacientes conocidos con diabetes mellitus tipos 2 que se someten a procedimiento quirúrgico bariátrico tienen una mejoría en su control glicémico, con una resolución del $85 \%$ de su control glicémico y reducción en el uso de fármacos diabetogénicos $(6,14)$.

\section{PROCEDIMIENTOS BARIÁTRICOS}

\section{Malabsortivo}

Los procedimientos de malabsorción comprenden la resección parcial del intestino delgado, que causa reducción del área de la mucosa intestinal lo que conduce a una menor absorción de nutrientes. Adicionalmente, estos procedimientos también restringen la ingesta calórica al limitar el tamaño de la cámara gástrica. Hasta el $85 \%$ de los pacientes con diabetes mellitus tipos 2 que se someten a este tipo de cirugía logran la remisión de la diabetes $(6,12)$.

\section{Bypass gástrico en $Y$ de Roux}

El bypass gástrico en $Y$ de Roux (RYGB), comúnmente conocido como cirugía de bypass gástrico, es el procedimiento más realizado y es el estándar de oro para ciruigía bariátirca. Este procedimiento 
comprende la construcción de una pequeña bolsa de estómago y una asa biliar, que conduce a ingesta calórica restringida y disminución de la absorción de nutrientes. El 95\% de la cámara gástrica, todo el duodeno y una parte del yeyuno se omiten del proceso gástrico (12).

Derivación biliopancreática con cruce $\underline{\text { duodenal }}$

La derivación biliopancreática con cruce duodenal consta de dos componentes: una gastrectomía parcial y un bypass intestinal. Se cree que este procedimiento preserva el proceso digestivo fisiológico $(6,12)$.

\section{$\underline{\text { Restrictivo }}$}

Los procedimientos restrictivos gástricos reducen la ingesta calórica y causan saciedad al limitar el volumen gástrico. Esto se puede hacer cambiando quirúrgicamente el tamaño del estómago como se hace en la gastrostomía en manga, la banda gástrica ajustable laparoscópica o la gastroplastia vertical. Estos procedimientos están asociados con aproximadamente el 46,2\% de la pérdida de exceso de peso corporal. Este tipo de cirugías ha demostrado conducir a la remisión de la diabetes mellitus tipo 2 en el $56,7 \%$ de los pacientes $(12,14)$.

\section{Banda gástrica ajustable laparoscópica}

Este procedimiento se caracteriza por la colocación de una banda ajustable alrededor del estómago proximal debajo de la unión gastroesofágica, formando una pequeña bolsa. Este procedimiento tiene menor morbilidad y mortalidad en comparación con otros procedimientos bariátricos, pero ha demostrado ser menos beneficioso que un procedimiento de malabsorción y se asocia con un mayor riesgo de reoperaciones (12).

\section{Gastrostomía en manga}

Comprende la resección parcial de la curvatura mayor del estómago. Este procedimiento es comparable a los procedimientos de malabsorción, pero ha demostrado tener un efecto mayor (6).

\section{COMPLICACIONES DE LA CIRUGÍA BARIÁTRICA}

Al aumentar la obesidad los procedimiento qurúrgicos relacionados con esta también van en alza. Se estima que en América, se realizaron 228.000 cirugías de adelgazamiento en 2017. De ellos, el 59\% fueron gastrectomía en manga, el 18\% fueron bypass gástrico, 3\% fueron banda gástrica y $1 \%$ derivación biliopancreática con cruce duodenal (15).

Las complicaciones posteriores al tratamiento quirúrgico de la obesidad grave varían según el procedimiento. realizado. Debido al alto volumen quirúrgico, mejorar la seguridad de estas operaciones se ha convertido en una alta prioridad, para una cirugía bariátrica segura y efectiva, y un control cuidadoso de resultados quirúrgicos $(14,15)$. La mortalidad asociada con los procedimientos bariátricos es del $0,28 \%$ a los 30 días después de la cirugía y del 0,35\% entre los 30 días y los 2 años después del procedimiento. Las complicaciones quirúrgicas mayores (es decir, TVP (trombosis venosa profunda), infección, fugas gástricas, fístulas, obstrucción del intestino delgado) se observan en el $5 \%$ al $40 \%$ de los casos y ocurren con mayor frecuencia en fumadores y diabéticos. La hipoglicemia reactiva es otra complicación observada con estos procedimientos, que se supone que se debe a una respuesta exagerada de la insulina posprandial al GLP1 (14).

e640 Revista Médica Sinergia Vol.6 (2), Febrero 2021 - ISSN:2215-4523 / e-ISSN:2215-5279 


\section{Distensión del remanente gástrico}

La distensión del remanente gástrico es una enfermedad rara pero potencialmente letal después de un bypass gástrico. El remanente gástrico es una bolsa ciega y puede distenderse si se produce íleo paralítico u obstrucción mecánica distal después de la operación. La lesión iatrogénica de las fibras vagales a lo largo de la curvatura menor también puede contribuir, posiblemente provocando un vaciado deficiente del estómago derivado. La distensión progresiva puede conducir en última instancia a rotura, derrame de contenido gástrico masivo y peritonitis grave subsiguiente (14).

Las características clínicas incluyen dolor, hipo, timpanismo en el cuadrante superior izquierdo, dolor de hombro, distensión, taquicardia o dificultad para respirar. La evaluación radiográfica puede demostrar una gran burbuja de aire gástrico. El tratamiento consiste en descompresión quirúrgica urgente con sonda de gastrostomía o gastrostomía percutánea. Se requieren exploración quirúrgica y descompresión inmediatas si el drenaje percutáneo no es factible o si se sospecha perforación (14).

\section{Estenosis del estoma}

Se ha descrito estenosis del estoma (anastomótica) en el 6-20\% de pacientes que se han sometido a cirugía bariátrica. La etiología es incierta, aunque la isquemia tisular, la úlcera marginal o el aumento de la tensión en la anastomosis gastroyeyunal desempeñan un papel (14).

Una estenosis del estoma se manifiesta clínicamente cuando la anastomosis se estrecha a un diámetro de $<10 \mathrm{~mm}$. Los pacientes suelen presentarse varias semanas después de la cirugía con náuseas, vómitos, disfagia, reflujo gastroesofágico y, finalmente, incapacidad para tolerar la ingesta oral, incluidos los líquidos. El diagnóstico suele establecerse mediante endoscopia o con una serie digestiva alta. La dilatación endoscópica con balón suele tener éxito $(14,15)$.

\section{Úlceras marginales}

Se han informado úlceras marginales en 0,6$16 \%$ de los pacientes. Las úlceras marginales ocurren cerca de la gastroyeyunostomía y son el resultado de la lesión del yeyuno con ácido, o pueden asociarse con una fístula gastrogástrica 0 , raramente, gastrocólica.

Las causas de las úlceras marginales incluyen:

1. Perfusión tisular deficiente debido a tensión o isquemia en la anastomosis.

2. Presencia de material extraño, como grapas o suturas no absorbibles

3. Exceso de exposición al ácido en la bolsa gástrica debido a fístulas gastrogástricas.

4. Uso de antiinflamatorios no esteroideos (AINES)

5. Infección por Helicobacter pylori

6. Fumado

Los pacientes con úlceras marginales pueden presentar náuseas, dolor abdominal, hemorragia gastrointestinal, estenosis del estoma o perforación. El diagnóstico de úlcera marginal se establece por endoscopia y el tratamiento médico inicial consiste en la supresión del ácido gástrico con un curso de seis semanas con inhibidores de bomba de protones, con o sin la adición de sucralfato, seguido de una endoscopia repetida para asegurar la curación. Durante el seguimiento, se deben suspender los 
antiinflamatorios no esteroideos, y se debe alentar a los pacientes a que dejen de fumar. Se debe realizar una tomografía computarizada (TAC) con contraste oral para descartar una fístula (14).

\section{$\underline{\text { Colelitiasis }}$}

La colelitiasis se desarrolla hasta en el $38 \%$ de los pacientes dentro de seis meses de cirugía. La pérdida de peso rapido también puede contribuir al desarrollo de cálculos biliares al aumentar la litogenicidad de la bilis. La alta frecuencia de presentación de la colelitiasis se puede reducir hasta un $2 \%$ con un curso de seis meses utilizando ácido ursodesoxicólico administrado de forma profiláctica después de la cirugía $(14,15)$.

\section{$\underline{\text { Hernia incisional ventral }}$}

Las hernias incisionales ventrales ocurren con una frecuencia de 0 a $1.8 \%$ en series laparoscópicas y tan alto como $24 \%$ en series abiertas. Las hernias incisionales se presentan con un bulto agrandado, dolor o síntomas obstructivos. Pacientes con obesidad severa está asociado con un aumento de la presión intraabdominal y, por lo tanto, un alto riesgo de desarrollo de hernia después de una laparotomía. Las indicaciones para la reparación quirúrgica temprana incluyen dolor significativo, obstrucción intestinal y rápido ensanchamiento de la hernia $(14,15)$.

\section{Obstrucción del intestino delgado}

La obstrucción del intestino delgado puede ocurrir en cualquier momento después de una cirugía bariátrica, con una incidencia de por vida del 3-5\%. Es causada comúnmente por una hernia del intestino delgado a través de uno de los defectos mesentéricos (es decir, hernias internas), también puede ser causada por enfermedad adhesiva, una hernia incisional o intususcepción (típicamente en la anastomosis yeyunoyeyunal). Los pacientes pueden presentarse de forma aguda o subaguda con síntomas vagos, intermitentes, espasmódicos y agudos.

Dolor abdominal generalmente no relacionado con la alimentación. Los pacientes sintomáticos deben someterse a un TAC y si esta es normal, pero los pacientes continúan con síntomas sugestivos de obstrucción intestinal, deben explorarse por vía laparoscópica para excluir una hernia interna $u$ otra causa de obstrucción $(14,15)$.

\section{Síndrome de dumping}

El síndrome de Dumping puede ocurrir en hasta el $50 \%$ de los casos postgástricos cuando se ingieren altos niveles de carbohidratos simples. Hay dos tipos de síndrome de dumping, temprano y tardío $(15,16)$.

- Síndrome de dumping temprano: tiene un inicio rápido, generalmente dentro de los 15 minutos. Es el resultado del rápido vaciado de alimentos en el intestino delgado. Debido a la hiperosmolalidad de los alimentos, se producen cambios rápidos de líquido desde el plasma al intestino, lo que resulta en hipotensión y una respuesta del sistema nervioso simpático. Los pacientes suelen presentar cólicos dolor abdominal, diarrea, náuseas y taquicardia (14-16). Los pacientes deben evitar los alimentos con alto contenido de azúcar simple y reemplazarlos con una dieta que consiste en alimentos ricos en fibra, carbohidratos complejos y proteínas. Modificación de comportamiento, como 
comidas pequeñas y frecuentes, y separar los sólidos de la ingesta de líquidos en 30 minutos. Por lo general, el dumping temprano es autolimitado y se resuelve en 7 a 12 semanas (14-16).

- Síndrome de Dumping tardío: actualmente denominado posprandial. La hipoglucemia hiperinsulinémica es una complicación poco común de la cirugía bariátrica. Ocurre en 0,1 a $0,3 \%$ de los pacientes, más comúnmente después de bypass gástrico en $Y$ de Roux. Síntomas incluyen: mareos, fatiga, diaforesis y debilidad, generalmente ocurren de una a tres horas después de la ingestión de una comida rica en carbohidratos y están asociados con hipoglucemia documentada (16-18).

La mayoría de los pacientes pueden tratarse con la misma modificación dietética. sugerido anteriormente para el síndrome de dumping temprano.

Los pacientes refractarios a la modificación de la dieta pueden ser tratado con medicamentos (p.ej. nifedipina, acarbosa, diazóxido u octreotido. El vaciado puede contribuir a la pérdida de peso en parte al hacer que el paciente modifique su alimentación (14-16).

\section{CONCLUSIÓN}

La obesidad no solo existe, sino que se promueve y exacerba con múltiples patologías y comorbilidades. El aumento de peso conduce a una mayor resistencia a la insulina, aumento de riesgo cardiovascular por enfermedades concomitantes como diabetes e hipertensión arterial. Por lo que es de importancia que todo profesional de salud conozca los aspectos generales de esta patología, se realice una captación a tiempo el paciente con sobrepeso y/u obesidad para realizar un abordaje temprano y preventivo en estos. Los cambios en estilo de vida son escenciales, sin embargo, la cirugía bariátrica demuestra ser superior a los métodos convencionales para una pérdida de peso duradera. También demostró tener un beneficio general en la reducción de la mortalidad en la obesidad severa y tiene efectos positivos sobre los parámetros metabólicos y las comorbilidades, como hipertensión, hiperlipidemia, síndrome metabólico, riesgo cardiovascular y control glucémico en diabetes.

\section{REFERENCIAS}

1. J Klish W, MD, A Skelton J, MD, MS, J Motil, K, MD, PhD, E Geffner, M, MD. (2020) Definition, epidemiology and etiology of obesity in children and adolescents. Jun, 2020, Jun 18, 2020. Uptodate Waltham, Massachusetts. Disponibe: https://www.uptodate.com/contents/definition-epidemiology-and-etiology-of-obesity-in-children-andadolescents

2. Upadhysy J, Farr O, Perakakis N, Ghaly W, Mantzoros C. Obesity as a Disease. Med Clin North Am, 2018;102(1):13-33.https://doi.org/10.1016/j.mcna.2017.08.004.

3. Arroyo-Johnson, Mincey K. Obesity epidemiology worldwide. Gastroenterol Clin North Am. 2016;45(4):571-579. https://doi.org/.1016/..gtc.2016.07.012.

4. Perreault L, MD, Pi-Sunyer, F, MD, MPH. Obesity in adultos: Prevalence, screening and evaluation. Mar 20, 2020, Jun, 2020. Uptodate Waltham, Massachusetts. Disponibe: https://www.uptodate.com/contents/obesity-in-adults- 
prevalence-screening-and-evaluation?search=obesity-in-adults-prevalence-screeningandevaluation\&source=search result\&selectedTitle $=1 \sim 150 \&$ usage type=default\&display rank=1

5. Bray MS, Loos RJ, McCaffery JM, et al. NIH working group report-using genomic information to guide weight management. Obesity (Silver Spring). 2016;24(1):14-22. https://doi.org/10.1002/oby.21381

6. Azim S, Kashyap R. Bariatric Surgery: Pathophysiology and Outcomes. Endocrinol Metab Clin North Am. 2016;45(4):905-921. https://doi.org/10.1016/i.ecl.2016.06.011.

7. Bjornland, T., Langaas, M., Grill, V., \& Mostad, I. L. (2017). Assessing gene-environment interaction effects of FTO, MC4R and lifestyle factors on obesity using an extreme phenotype sampling design: Results from the HUNT study. PLoS One. 2017;12(4):e0175071. https://doi.org/10.1371/journal.pone.0175071.

8. Farr, O. M., Tsoukas, M. A., \& Mantzoros, C. S. (2015). Leptin and the brain: Influences on brain development, cognitive functioning and psychiatric disorders. Metabolism. 2015;64(1):114-30. https://doi.org/10.1016/j.metabol.2014.07.004.

9. Doolan, K. J., Breslin, G., Hanna, D., \& Gallagher, A. M. (2015). Attentional bias to food-related visual cues: is there a role in obesity?. Proc Nutr Soc. 2015;74(1):37-45. https://doi.org/10.1017/S002966511400144X.

10. Sahakyan, K. R., Somers, V. K., Rodriguez-Escudero, J. P., Hodge, D. O., Carter, R. E., Sochor, O., ... LopezJimenez, F. (2015). Normal-Weight Central Obesity: Implications for Total and Cardiovascular Mortality. Ann Intern Med. 2015;163(11):827-35. https://doi.org/10.7326/M14-2525.

11. Skinner A. C., Perrin, E. M., Moss, L. A., \& Skelton, J. A. (2015). Cardiometabolic Risks and Severity of Obesity in Children and Young Adults. N Engl J Med. $2015 ; 373(14): 1307-17$. https://doi.org/10.1056/NEJMoa1502821.

12. Angrisani, L., Santonicola, A., lovino, P., Formisano, G., Buchwald, H., \& Scopinaro, N. (2015). Bariatric Surgery Worldwide 2013. Obes Surg. 2015;25(10):1822-32. https://doi.org/10.1007/s11695-015-1657-z

13. Seravalle, G., \& Grassi, G. (2017). Obesity and hypertension. Pharmacol Res. 2017;122:1-7. https://doi.org/10.1016/j.phrs.2017.05.013

14. Ellsmere, J. Late complications of bariatric surgical operations. Wolters Klumer: Kunnis L; Aug 01, 2020. https://www.uptodate.com/contents/late-complications-of-bariatric-surgical-operations

15. Kassir, R., Debs, T., Blanc, P., Gugenheim, J., Ben Amor, I., Boutet, C., \& Tiffet, O. (2016). Complications of bariatric surgery: Presentation and emergency management. Int $J$ Surg. 2016; 27:77-81. https://doi.org/10.1016/j.ijsu.2016.01.067

16. Chaves, y. Da S, \& Destefani, A. C. (2016). Pathophysiology, diagnosis and treatment of dumping syndrome and its relation to bariatric surgery. Arq Bras Cir Dig. 2016; 29Suppl 1(Suppl 1):116-119.https://doi.org/10.1590/0102$\underline{6720201600 \text { s10028 }}$ 